\title{
Information and analytical support for the detection and investigation of crimes
}

\author{
Olga Borisovna Dronova ${ }^{1 *}$, Aleksey Aleksandrovich Kurin ${ }^{1}$, Anna Pavlovna Alekseeva ${ }^{2}$, \\ Dmitriy Nikolaevich Sidorenko ${ }^{1}$, and Svetlana Dmitrievna Guseva ${ }^{1}$ \\ ${ }^{1}$ Volgograd Academy of the Ministry of Internal Affairs of Russia, Volgograd, Russia \\ ${ }^{2}$ Kaliningrad branch of the Saint Petersburg University of the Ministry of Interior Affairs of Russia, \\ Kaliningrad, Russia
}

\begin{abstract}
The effectiveness of law enforcement agencies is constantly monitored by the public, and its indicator is the safety of citizens, the ability to exercise their rights and freedoms. One of the strategic resources of the life of modern society is various information. Computer networks, geographical information systems, means of tactical forecasting of crimes, numerous integrated databases are already widely applied in the activities of the law enforcement agencies. Despite this, the main information resource for forensic purposes represented by the information arrays of the forensic registration system still remains a repository of heterogeneous data, the collective use of which causes significant organizational and technical difficulties. Information and analytical support for the disclosure and investigation of crimes is aimed at obtaining an analytical information product about the structure, hierarchy, and composition of criminal groups, about the commodity and cash flows and relations of individuals and legal entities, about their joint criminal activities, about the unidentified circumstances of the events of the crime to be proven. The implementation of cumulative inspections of objects and traces on various information arrays helps to establish the missing information about the crime event.
\end{abstract}

Keywords: forensic registration, telecommunications systems, criminally significant information, spatial and temporal connections, forensic analysis

\section{Introduction}

The success of the police largely depends on the effectiveness of information and analytical support for the processes of detection, disclosure, and investigation of crimes. In order to obtain and process the necessary data, law enforcement entities use information systems and hardware and software complexes. Currently, when working with the information resources of the internal affairs, the emphasis is shifting from obtaining mainly reference data to the results of their analytical processing to obtain relevant forensic and operationally relevant information. The implementation of the concept of creating a unified system of information and analytical support for the activities of the law enforcement agencies of the Russian Federation has not fully solved the problems associated with the lack of information support.

\footnotetext{
*Corresponding author: nio-va@rambler.ru
} 
It should be mentioned that analytical processing of documentary and actual data about the crime event is necessary to obtain directory and investigative information. Information and analytical support for the detection and investigation of crimes is aimed at obtaining an analytical information product [1]. The processed information may contain data on the structure, hierarchy, and composition of criminal groups, information on commodity and cash flows transferred by individuals and legal entities, their personal data, movements, contacts, vehicles, firearms, mobile communication devices used by members of criminal groups and their contacts, as well as other previously unknown circumstances of the crime [2].

Information and analytical support represent a distributed collection of heterogeneous data, their extraction, transformation, loading into the system, storage, and analysis [3, 4]. The main advantage of analytical data processing is the ability to perform model calculations on them in order to obtain new output data presented in a visual structured form (a multidimensional scheme, a graphical chart of connections of a person of interest, objects, and traces). For the implementation of analytical measures, several conditions shall be complied with [5]: the presence of integrated information arrays; the presence of a telecommunications environment; the availability of tools for analytical data processing.

\section{Methods}

One of the main information resources of law enforcement agencies is the system of forensic registration. However, due to a number of shortcomings in its functioning, expedient and promising way of integration of criminally significant information would be the creation of a single telecommunications system of forensic registration as a conceptual platform that determines the key directions of its development.

The concept of a unified telecommunications system for forensic registration provides:

- integration of heterogeneous arrays at the intradepartmental and interdepartmental levels in the format of the functioning of the unified information interaction system;

- monitoring support for access to the telecommunications infrastructure of accounting and registration data;

- intelligent, analytical processing of integrated accounting and registration data, generation of an analytical product.

\section{Results and discussion}

After working through the issue of the specifics of information interaction, it has been concluded that it is advisable to provide access to the unified telecommunications system of forensic registration to departments that provide operational support for the investigation of crimes and to those who provide their information support [6].

To increase the effectiveness of information and analytical support, in addition to the mentioned registration objects, it is necessary to use information from online civil resources that are not related to criminal activities and require its combination with operational information. Thus, predictive analytics involves automated algorithms for accessing operational-search resources, processing secret-service messages, operational reports, and background information that circulate in network resources and social telecommunications networks. The purpose of this analysis is to identify persons with a certain criminal potential, which is calculated in terms of probability theory and mathematical statistics.

To obtain complete data, specialized information arrays of units to combat terrorism, extremism, drug trafficking, and Interpol resources shall be used. This information, supplemented with information obtained from open segments of the Internet, and compared 
with information from the unified telecommunications system of forensic registration, allows determining personal qualities, interests, criminal potential, environment, connections, network and mobile communications with accomplices, ideological leaders, organizers of criminal activities.

In addition, there should be created an array of references to reference and auxiliary records (photo libraries, reference books) of firearms and cold weapons and traces of their use, types of shoes, vehicle tire treads, tools for various purposes. Databases of reference information about objects can be imported into the analytical module of the forensic registration system from the photo libraries of field funds and reference and auxiliary records of forensic units.

A significant reserve for the unified telecommunications system of forensic registration is the possibility of implementing monitoring technologies. It is advisable to obtain information (geolocation, spatial-temporal nature, metadata) through technical wireless communication channels included in a single telecommunications complex by means of a system of transponders, satellite communication sensors, and unified readers [7]. The inclusion of such information in the system of forensic registration seems appropriate from the point of view of establishing a connection between the object (subject) and the criminal event.

With regard to the fact that the use of the resources of the system of forensic registration is classified as law enforcement intelligence operations, to implement its capabilities during law enforcement intelligence operations, it is advisable to enter into the accounting and registration arrays data on mobile telecommunications systems (radio frequency identification devices, satellite navigation systems), which are equipped with many objects of registration. This category may include firearms, explosives, and industrial explosive devices, a number of licensed objects of the consumer market, items of historical and cultural value, as well as other objects of accounting and registration activities.

To optimize the control over objects of operational interest, it is advisable to consider their marking using bar codes, QR-codes, or using radio-frequency RFID identifiers. The forensic registration system shall support the requirements for monitoring the current location and the sequence of movement of the monitored objects. Sensors determine their location through the global positioning system, periodically, send data about their position via Google Earth or Google.

The advantages of integrated registration data in combination with mobile telecommunications are revealed during the analytical processing of information arrays of the forensic registration system. The use of cluster analysis methods [8] helps to establish links between several crime events through the similarity of traces, objects of criminal encroachment, and persons involved. As soon as the objects of the cluster of interest are established in the circumstances of other crime events, a link between criminal cases will be created automatically.

A promising direction is the use of a forensic registration system for case profiling. The characteristics of specific situations may be used for grouping events of crimes and identifying trends, such as the type of attacks, a group of persons, signs of crimes committed by professionally trained individuals, the value of the stolen property, etc. The profiler [9, 10] searches for options with regard to the circumstances and signs of the crime event. Each feature can be considered according to its significance coefficient.

The system's analytical functions will ensure that relationships and coincidences between events and objects are detected in the information system. For example, vehicle registration numbers and DNA profiles found at various crime scenes can be automatically linked by the system based on matches of other registration objects. Information about such events can be presented on electronic maps of the area, diagrams, or graphical charts that represent connections through applications such as MS Visio or in an IBM Analyst notebook [11]. The 
diagrams may reflect links between tracks, objects, and suspects in one or more criminal cases. This processing is performed by means of data mining [12], and the result is visualized, indicating an implicit relationship between events, objects, and persons.

Using this module, statistical information can be systematized and presented on a graphical chart in retrospect. The forensic registration system can be integrated with other applications that allow creating statistical and analytical reports, as well as export information to various types of software and interagency systems. Office tablets (computers) allow the creation of reviews, reports, charts, and notes in the following applications: Microsoft Word, Microsoft Excel, Microsoft Visio, and IBM Analyst. In addition to connections with wellknown software applications [13], it is also possible to connect to the back-office systems of police units.

\section{Conclusion}

The results of analytical processing may be sent to the operational units, both on an initiative basis and by the initiators of requests. In this case, the analytical materials are used as the basis for preventive measures $[3,14]$. Thus, global machine analysis technologies contribute to the planning and implementation of tactical operations aimed at detecting and countering crimes.

The current level of technical development of the society proves that there are all conditions for creating a unified system for obtaining analytical information when using telecommunications, geoinformation, and navigation systems, including information security technologies, artificial intelligence technologies.

\section{References}

1. O.B. Dronova, A.A. Kurin, Rus. J. Crim. 11(2), 318-326 (2017)

https://doi.org/10.17150/2500- 4255.2017.11(2).318-326.

2. O.P. Gribunov, Bul. Tomsk State Univ. 446, 207-211 (2019) https://doi.org/10.17223/15617793/446/27

3. M. Gentili, P.B. Mirchandani, App. Loc. Anal. 232, 307-327 (2015). https://doi.org/10.1007/978-3-319-20282-2_13

4. A.N. Lakhin, Pub. Safety, Leg. Law and Order in the III Mil. 3-3, 290-294 (2017)

5. A.B. Tambawal, R.M. Noor, R. Salleh, C. Chembe, M.H. Anisi, O. Michael, J. Lloret, Telecommunication Systems, 70(4), 595-616 (2019). https://doi.org/10.1007/s11235018-00542-8

6. O.B. Dronova, B.P. Smagorinskiy, V. Yastrebov, Counteraction to E-Commerce Crimes Committed with the Use of Online Stores, in Studies in Systems, Decision and Control, 181, 121-131 (2019) https://doi.org/10.1007/978-3-030-01358-5_12

7. A.A. Kurin, S.M. Kolotushkin, Forensic exam. 4, 123-131 (2018)

8. H.F. Bermudez, R. Sanchez-Iborra, J.L. Arciniegas, W.Y. Campo, M.-D. Cano, Telecommunication Systems, 71(3), 491-504 (2019). https://doi.org/10.1007/s11235018-0521-6

9. R. Figueiredo, A, Zúquete, T. Oliveira e Silva, Massively Parallel Identification of Privacy-Preserving Vehicle RFID Tags, in Lecture Notes in Computer Science, 8651, 36-53 (2014). https://doi.org/10.1007/978-3-319-13066-8_3

10. M.S. Kolosovich, State and Law, 7, 16-28 (2019) https://doi.org/10.31857/S013207690005875-4

11. S. Enchelmaier, Restrictions on the Use of Goods and Services, in The Reach of Free Movement, 85-108 (T.M.C. Asser Press, The Hague, 2017) 
12. N.V. Pavlichenko, A.I. Tambovtsev, Proc. Acad. of Management Min. of Internal Aff. Rus. 2(54), 62-68 (2020)

13. S. Faruque, Radio Frequency Multiple Access Techniques Made Easy (Springer International Publishing, New York, 2019)

14. A.L. Osipenko, Soc. and Law. 4(66), 80-87 (2018) 